DRAFT VERSION MARCH 6, 2022

Preprint typeset using LTEX style emulateapj v. 11/10/09

\title{
PROBING FUNDAMENTAL CONSTANT EVOLUTION WITH NEUTRAL ATOMIC GAS LINES
}

\author{
N. Kanekar ${ }^{1,2}$, J. X. Prochaska ${ }^{3}$, S. L. Ellison ${ }^{4}$, J. N. Chengalur ${ }^{1}$ \\ Draft version March 6, 2022
}

\begin{abstract}
We have detected narrow HI $21 \mathrm{~cm}$ and CI absorption at $z \sim 1.4-1.6$ towards Q0458-020 and Q2337-011, and use these lines to test for possible changes in the fine structure constant $\alpha$, the proton-electron mass ratio $\mu$, and the proton gyromagnetic ratio $g_{p}$. A comparison between the HI $21 \mathrm{~cm}$ and CI line redshifts yields $\Delta X / X=[+6.8 \pm 1.0] \times 10^{-6}$ over $0<\langle z\rangle<1.46$, where $X=g_{p} \alpha^{2} / \mu$, and the errors are purely statistical, from the gaussian fits. The simple line profiles and the high sensitivity of the spectra imply that statistical errors in this comparison are an order of magnitude lower than in previous studies. Further, the CI lines arise in cold neutral gas that also gives rise to $\mathrm{HI} 21 \mathrm{~cm}$ absorption, and both background quasars are core-dominated, reducing the likelihood of systematic errors due to local velocity offsets between the hyperfine and resonance lines. The dominant source of systematic error lies in the absolute wavelength calibration of the optical spectra, which appears uncertain to $\sim 2 \mathrm{~km} / \mathrm{s}$, yielding a maximum error in $\Delta X / X$ of $\sim 6.7 \times 10^{-6}$. Including this, we obtain $\Delta X / X=[+6.8 \pm 1.0$ (statistical $) \pm 6.7$ (max.systematic $)] \times 10^{-6}$ over $0<\langle z\rangle<1.46$. Using literature constraints on $\Delta \mu / \mu$, this is inconsistent with claims of a smaller value of $\alpha$ from the many-multiplet method, unless fractional changes in $g_{p}$ are larger than those in $\alpha$ and $\mu$.
\end{abstract}

Subject headings: atomic processes — galaxies: high-redshift — quasars: absorption lines

\section{INTRODUCTION}

A critical assumption in the standard model of particle physics is that low-energy coupling constants and particle masses do not vary with space or time. This assumption breaks down in most theories that attempt to unify the standard model and general relativity (e.g. Marciano 1984). The detection of such spatio-temporal variation in coupling constants like the fine structure constant $\alpha$, or the ratios of particle masses (e.g. the proton-electron mass ratio $\mu \equiv m_{p} / m_{e}$ ), would imply new physics beyond the standard model and is hence of great interest (e.g. Uzan 2003).

Comparisons between the redshifts of spectral lines detected in distant galaxies provide an important tool to probe changes in $\alpha, \mu$ and the proton gyromagnetic ratio $g_{p}$ over cosmological times (e.g. Wolfe et al. 1976; Dzuba et al. 1999; Chengalur \& Kanekar 2003; Flambaum \& Kozlov 2007). Most such studies have yielded constraints on changes in $\alpha, \mu$ and $g_{p}$, with different systematic effects [see Kanekar (2008) for a recent review]. At present, the only technique that has found statistically-significant evidence for a change in one of the fundamental constants is the "many-multiplet" method (Dzuba et al. 1999). Murphy et al. (2004) obtained $[\Delta \alpha / \alpha]=$ $(-5.7 \pm 1.1) \times 10^{-6}$ from Keck High Resolution Ultraviolet Echelle Spectrometer (HIRES) optical spectra of $143 \mathrm{ab}$ sorbers with a mean redshift $\langle z\rangle=1.75$, suggesting that $\alpha$ was smaller at earlier times. Other studies, applying a similar technique to Very Large Telescope (VLT) data on smaller samples, have not confirmed this result (e.g. Levshakov et al. 2006; Srianand et al. 2007). However, Murphy et al. (2008b) argue that the errors in these studies have been under-estimated, and

\footnotetext{
${ }^{1}$ National Centre for Radio Astrophysics, TIFR, Ganeshkhind, Pune 411007, India

${ }^{2}$ National Radio Astronomy Observatory, 1003 Lopezville Road, Socorro, NM87801, USA; nkanekar@nrao.edu

${ }^{3}$ UCO/Lick Observatory, UC Santa Cruz, Santa Cruz, CA 95064, USA

${ }^{4}$ Department of Physics and Astronomy, University of Victoria, Victoria, B.C., V8P 1A1, Canada
}

that results from small samples are more prone to systematic effects related to the fitting of spectral components to complex absorption profiles. In the case of $\mu$, King et al. (2008) used VLT Ultraviolet Echelle Spectrograph (UVES) spectra of ro-vibrational $\mathrm{H}_{2}$ lines in three damped Ly $\alpha$ systems at $z \sim 2.6-3$ to find $[\Delta \mu / \mu]=(-2.6 \pm 3.0) \times 10^{-6}$. Note that none of the above error estimates include recently-detected systematic effects due to distortions in the wavelength scales of the HIRES and UVES spectrographs (e.g. Griest et al. 2010). Finally, constraints on changes in $\alpha, \mu$ and $g_{p}$ have also been obtained from radio techniques (e.g. Carilli et al. 2000; Kanekar et al. 2004; Kanekar et al. 2005; Murphy et al. 2008a), although at lower redshifts.

Comparisons between the redshifts of Hi $21 \mathrm{~cm}$ (hyperfine) and ultraviolet resonance dipole transitions are sensitive to changes in $X \equiv g_{p} \alpha^{2} / \mu$ (Wolfe et al. 1976). The best resonance lines for this method are those arising from neutral atomic species (e.g. CI, FeI, etc), as these species are most likely to be physically associated with the HI. The CI multiplets are likely to be the best among the neutral resonance transitions as they typically arise in cold gas which also gives rise to the HI $21 \mathrm{~cm}$ absorption (e.g. Jenkins \& Tripp 2001; Srianand et al. 2005). The ionization potentials of $\mathrm{CI}$ and $\mathrm{HI}$ are also similar, $11.3 \mathrm{eV}$ for $\mathrm{CI}$ and $13.6 \mathrm{eV}$ for $\mathrm{HI}$ (cf. MgI, which is more easily detectable than $\mathrm{CI}$ in high- $z$ absorbers, has an ionization potential of $7.6 \mathrm{eV}$, as well as a high dielectronic recombination rate that can give significant $\mathrm{MgI}$ absorption in warm, ionized gas; Pettini et al. 1977). Finally, absorbers with a single (or dominant) spectral component in both $\mathrm{HI} 21 \mathrm{~cm}$ and CI transitions are best-suited for such studies.

HI $21 \mathrm{~cm}$ and CI absorption have hitherto both been detected in only one high- $z$ absorber, the $z \sim 1.776$ system towards $1331+170$, yielding a weak constraint on changes in $X \equiv g_{p} \alpha^{2} / \mu$ (Cowie \& Songaila 1995). However, the CI line in this absorber has two clear spectral components (Dessauges-Zavadsky et al. 2004), implying ambiguities (and thus, large systematic errors) in the comparison with the 
HI $21 \mathrm{~cm}$ line. In this Letter, we report the detection of narrow, single-component $\mathrm{HI} 21 \mathrm{~cm}$ and $\mathrm{CI}$ absorption in two absorbers at $z \sim 1.4-1.6$, that allow a high-sensitivity study of changes in the fundamental constants.

\section{SPECTRA AND RESULTS}

The Giant Metrewave Radio Telescope (GMRT) and the Green Bank Telescope (GBT) were used to detect HI $21 \mathrm{~cm}$ absorption at $z \sim 1.3609$ towards Q2337-011 and $z \sim 1.5605$ towards Q0458-020, respectively, in a survey for HI $21 \mathrm{~cm}$ absorption in strong MgII absorbers [GMRT proposals 7NKa02, $10 \mathrm{NKa} 02$, and GBT proposal $6 \mathrm{~A}-026$; see Kanekar et al. (2009) for details]. The HI $21 \mathrm{~cm}$ spectra are shown in the lower panels of Figure 1. The root-mean-square (RMS) noise values, measured from off-line regions in the spectra, are $\sim 0.03$ per $1.9 \mathrm{~km} / \mathrm{s}$ channel (Q2337-011) and $\sim 0.0037$ per $1.3 \mathrm{~km} / \mathrm{s}$ channel (Q0458-020), in optical depth units.

We then carried out a search for redshifted CI absorption from the two absorbers, using the high-sensitivity Keck-HIRES spectrum of Prochaska \& Wolfe (1997) towards Q0458-020 (observed on 31 October and 1 November 1995), and a new HIRES spectrum of Q2337-011 (observed on 18 and 19 September 2006). This resulted in the detection of the CI $\lambda 1560$ and CI $\lambda 1657$ transitions at $z \sim 1.3609$ towards Q2337-011, and the $\mathrm{CI} \lambda 1560$ and $\mathrm{CI}^{*} \lambda 1561$ transitions at $z \sim 1.5605$ towards Q0458-020. Of these, the CI $\lambda 1560$ line towards Q2337-011 is blended with another line, while the $\mathrm{CI}^{*} \lambda 1561$ line towards Q0458-020 is detected at low significance; these will hence not be used in the later analysis. The CI $\lambda 1657$ line towards Q2337-011 and the CI $\lambda 1560$ line towards Q0458-020 are shown in the upper panels of Figure 11 The signal-to-noise ratios per pixel are $\sim 25$ at a resolution of $R \sim 50000$ (Q2337-011), and $\sim 15$ at $R \sim 37000$ (Q0458-020), in the vicinity of the above CI transitions.

For all spectra, the RMS noise was measured from absorption-free spectral regions around the line in question. A single-gaussian model was then used to independently fit each of the 1-D spectra in the Hi $21 \mathrm{~cm}$ and Ci lines. This model yielded an excellent fit to all spectra, with reduced chi-square values $<1.06$ in all cases, and no evidence for statisticallysignificant features in the residual spectra. A KolmogorovSmirnov rank-1 test found all residual 1-D spectra to be consistent (within $1.1 \sigma$ significance) with being drawn from a normal distribution. The RMS noise values used for the spectral fits were scaled (marginally) to obtain $\chi^{2}=1$ in all cases; the resulting fits were then used to measure the peak absorption redshift for each transition. These redshifts are listed in columns (2) and (3) of Table 1, note that the CI $\lambda 1560$ and $\mathrm{CI} \lambda 1657$ lines have laboratory vacuum wavelengths of 1560.3092 $\AA$ and 1657.9283 A, respectively (Morton 2003), while the HI $21 \mathrm{~cm}$ line frequency is 1420.405751766 (1) $\mathrm{MHz}$ (Essen et al. 1971).

A useful test of the limiting accuracy in such fits can be obtained by combining the minimum uncertainties contributed by all line pixels to determine the best velocity accuracy that might be obtained with a given spectral fit (Murphy et al. $2008 \mathrm{~b}$ ). In all cases, the redshift errors in Table 1 are larger than this limiting accuracy (by factors $\lesssim 2$ ), as expected for real spectra. We also tested that adding additional components to the fits does not significantly alter the results.

Assuming that the $\mathrm{HI} 21 \mathrm{~cm}$ and $\mathrm{CI}$ lines arise in the same gas, the fractional change in $X \equiv g_{p} \alpha^{2} / \mu$ is related to the observed HI $21 \mathrm{~cm}$ and $\mathrm{CI}$ redshifts by $\Delta X / X=\left[z_{\mathrm{CI}}-\right.$
TABLE 1

PARAMETERS OF THE SINGLE-GAUSSIAN FITS TO THE HI $21 \mathrm{CM}$ AND CI SPECTRA TOWARDS Q2337-011 AND Q0458-020.

\begin{tabular}{|c|c|c|c|c|}
\hline & $z_{21 \mathrm{~cm}}$ & $z_{\mathrm{CI}}$ & $\begin{array}{c}\Delta V^{\dagger} \\
\mathrm{km} / \mathrm{s}\end{array}$ & $\begin{array}{c}\Delta X / X \\
\times 10^{-6}\end{array}$ \\
\hline Q2337-011 & $1.3608644(13)$ & $1.3608801(15)$ & $1.99 \pm 0.25$ & $+6.64 \pm 0.84$ \\
Q0458-020 & $1.5605300(25)$ & $1.5605480(38)$ & $2.11 \pm 0.53$ & \begin{tabular}{c}
$+7.0 \pm 1.8$ \\
\hline
\end{tabular}
\end{tabular}

$\dagger$ The velocity offset of the Cr line from the HI $21 \mathrm{~cm}$ line.

$\left.z_{21 \mathrm{~cm}}\right] /[1+\bar{z}]$, where $\bar{z}=\left(z_{\mathrm{CI}}-z_{21 \mathrm{~cm}}\right) / 2$ (Wolfe et al. 1976). This yields $\Delta X / X=(+6.64 \pm 0.84) \times 10^{-6}$ for the $z \sim 1.3609$ absorber towards Q2337-011 and $\Delta X / X=(+7.0 \pm 1.8) \times$ $10^{-6}$ for the $z \sim 1.5605$ absorber towards Q0458-020. Averaging these values (with equal weights) gives the result $\Delta X / X=(+6.8 \pm 1.0) \times 10^{-6}$, over $0<z<1.46$. We emphasize that the errors quoted here are purely statistical ones, from the fits; systematic effects are discussed in the next section.

\section{SYSTEMATIC EFFECTS}

At the outset, it should be emphasized that, unlike the many-multiplet method which only requires accurate relative wavelength calibration between different lines in the same optical spectrum, the comparison between HI and CI lines requires accurate absolute wavelength calibration of the optical spectra. On the other hand, the many-multiplet method is based on a first-order effect (the wavelengths of transitions used in this analysis have the same zeroth-order dependence on $\alpha$ ), while the present method uses the zeroth-order dependences of the hyperfine and resonance line frequencies on $\alpha$, $\mu$ and $g_{p}$. This implies that systematic effects (e.g. due to wavelength mis-calibration) are less important by an order of magnitude in the hyperfine/resonance comparison than in the many-multiplet analysis. For example, a velocity uncertainty of $0.3 \mathrm{~km} / \mathrm{s}$ implies an error of $[\Delta \alpha / \alpha]=10^{-5}$ in the manymultiplet method (Murphy et al. 2001), but of $\Delta X / X=10^{-6}$ in the hyperfine/resonance comparison.

The CI lines in both absorbers are at higher redshifts than the HI $21 \mathrm{~cm}$ lines, by $\sim 2 \mathrm{~km} / \mathrm{s}$. Systematic effects that might cause such a velocity offset include (1) different relative isotopic abundances at $z \sim 1.5$ from the local Universe, that might cause the rest $\mathrm{CI}$ wavelengths in the two absorbers to be higher than their laboratory values, (2) errors in the absolute wavelength calibration of the optical spectra, and (3) "local" velocity offsets between the $\mathrm{CI}$ and $\mathrm{HI} 21 \mathrm{~cm}$ lines in the two absorbers. Note that frequency mis-calibration of the $\mathrm{HI} 21 \mathrm{~cm}$ spectra is unlikely to be the source of such errors, as the absolute frequency scale here is set by the accuracy of masers and local oscillators (typically $<10 \mathrm{~Hz}$, two orders of magnitude lower than the observed velocity offset between the $\mathrm{HI} 21 \mathrm{~cm}$ and $\mathrm{CI}$ lines).

The velocity shifts of the ${ }^{14} \mathrm{C}$ and ${ }^{13} \mathrm{C}$ isotopic transitions relative to the main ${ }^{12} \mathrm{C}$ isotopic lines are, respectively, $+0.46 \mathrm{~km} / \mathrm{s}$ and $+0.84 \mathrm{~km} / \mathrm{s}$ for the CI $\lambda 1657$ line, and $-3.29 \mathrm{~km} / \mathrm{s}$ and $-6.10 \mathrm{~km} / \mathrm{s}$ for the CI $\lambda 1560$ line (Berengut et al. 2006). The isotopic velocity shift is clearly too small to account for the observed velocity offset in the case of the CI $\lambda 1657$ line. Conversely, in the case of the CI $\lambda 1560$ line, the isotopic shift yields the opposite sign from the observed velocity offset between CI and Hi lines. We can thus rule out the hypothesis that differing carbon isotopic abundances (compared to Galactic values) might account for 

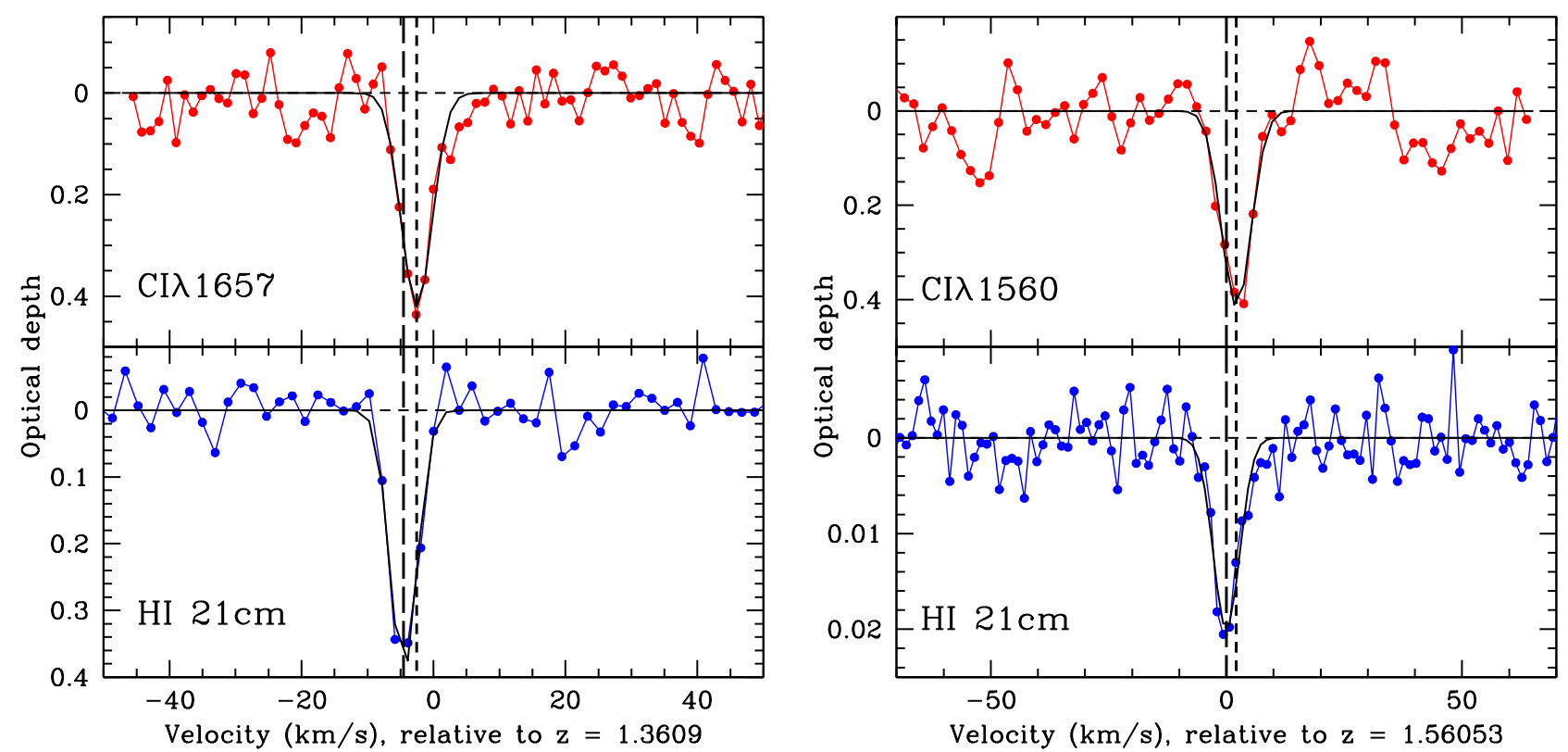

FIG. 1.- HI 21 cm and CI spectra towards [A] Q2337-011 and [B] Q0458-020. In each panel, the solid line shows the 1-gaussian fit to the spectrum. The small-dashed and large-dashed vertical lines indicate the CI and HI $21 \mathrm{~cm}$ redshifts, respectively; the CI redshift is seen to be higher than the HI $21 \mathrm{~cm}$ redshift in both panels.

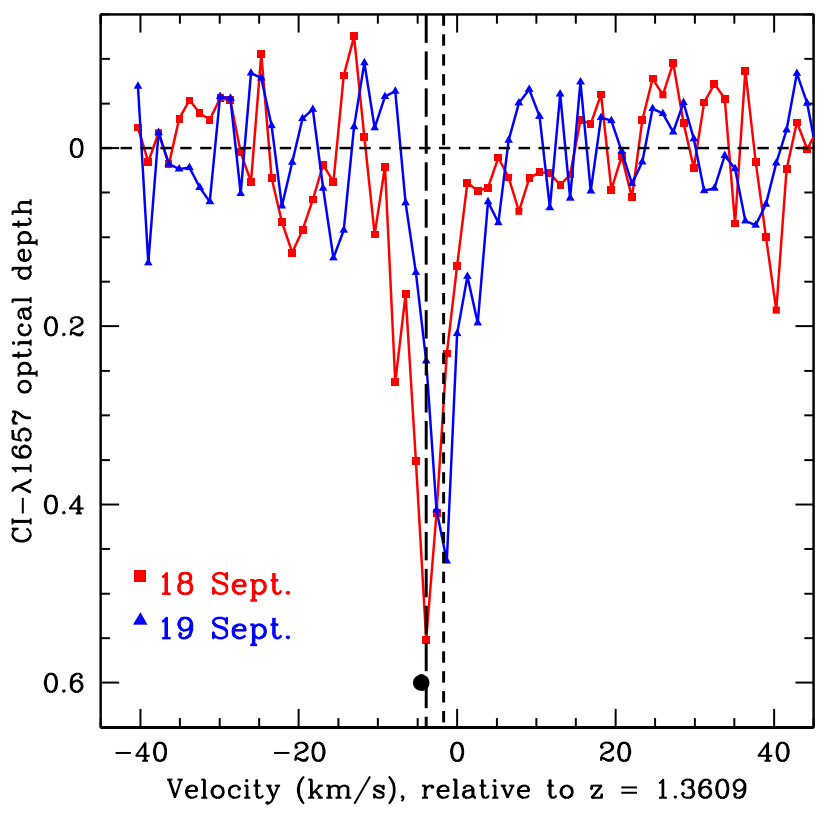

FIG. 2.- A comparison between the CI $\lambda 1657$ line profiles obtained on 18 and 19 September, plotted as squares and triangles, respectively. The dashed vertical lines indicate the CI redshifts obtained from 1-gaussian fits to each spectrum; these are offset from each other by $\sim 2 \mathrm{~km} / \mathrm{s}$. The solid circle shows the HI $21 \mathrm{~cm}$ redshift; see main text for discussion.

the observed velocity offset between the HI $21 \mathrm{~cm}$ and CI transitions.

Mis-calibration of the absolute wavelength scale of the optical spectra could arise from a number of causes (e.g. Murphy et al. 2001). In particular, the spectrum towards Q0458-020 was obtained in 1995, before Keck-HIRES was fitted with an image rotator. It was thus not possible to hold the slit perpendicular to the horizon during these observations, implying that atmospheric dispersion across the slit could pro- duce errors in the wavelength scale (Murphy et al. 2001). Further, while the data towards Q2337-011 were obtained in 2007, with the use of the image rotator, Griest et al. (2010) have found evidence for drifts in the Keck-HIRES wavelength scale with time, with amplitudes of $\sim 2 \mathrm{~km} / \mathrm{s}$ over multiple observing epochs. The source of these velocity drifts is still unclear, although temperature changes, changes in the position of the quasar on the slit, as well as physical shifts in the echelle grating or cross-disperser are all possible causes.

To test for systematic effects in the absolute wavelength calibration of the optical spectra, we analysed archival KeckHIRES data towards 0458-020 from 5 and 6 October 2004 (obtained using the image rotator). While this spectrum has lower S/N than the 1995 spectrum (as the opticallyvariable quasar was in a fainter state in 2004), the unsaturated NiII $\lambda 1317$ transition from a higher-redshift $(z=2.03945)$ damped Lyman- $\alpha$ system is clearly detected in both spectra; this line lies at $\sim 4003 \AA$, within $10 \AA$ of the CI $\lambda 1560$ line shown in Figure 1B]. Cross-correlating the NiII lines detected in the two HIRES spectra yielded a cross-correlation peak at a velocity offset of $+1.60 \pm 0.84 \mathrm{~km} / \mathrm{s}$; the error was determined by cross-correlating 10000 pairs of simulated spectra with the shape and noise properties of the actual spectra. If this velocity offset between the NiII lines arises due to atmospheric dispersion across the slit during the $1995 \mathrm{ob}-$ servations, it would imply that the CI line redshift has been under-estimated in the 1995 spectrum. Correcting for this effect would increase the redshift offset between the $\mathrm{HI} 21 \mathrm{~cm}$ and $C$ I lines.

We also separately analysed the Keck-HIRES data towards Q2337-011 from 18 and 19 September 2006, to test whether the same line redshift was obtained from the two runs. Figure 2 shows the CI $\lambda 1657$ line profiles obtained on 18 and 19 September; these are clearly offset from each other. A single-component gaussian fit was used to measure the $\mathrm{CI} \lambda 1657$ redshift from each spectrum; the offset between the $\mathrm{CI} \lambda 1657$ redshifts is $2.15 \pm 0.44 \mathrm{~km} / \mathrm{s}$. Similar velocity 
offsets $(\sim 2 \mathrm{~km} / \mathrm{s})$ were seen between other lines in the two spectra. Note that a single ThAr lamp exposure was used to calibrate the two science exposures on Q2337-011 on each day; the ThAr exposure was taken immediately before/after the science exposures. Interestingly, the CI $\lambda 1657$ redshift of 18 September $\left[z_{\mathrm{CI}}=1.3608694(26)\right]$ is in reasonable agreement with the $\mathrm{HI} 21 \mathrm{~cm}$ redshift $\left[z_{21} \mathrm{~cm}=1.3608644(13)\right]$, indicated by the solid circle in Figure 2, the redshift offset between the HI $21 \mathrm{~cm}$ and $\mathrm{CI}$ lines is dominated by the CI data from 19 September. The offsets of $\sim 2 \mathrm{~km} / \mathrm{s}$ between our two observing epochs are consistent with the velocity drifts in the HIRES wavelength scale found by Griest et al. (2010) (see their Figure 5). We hence conclude that the absolute wavelength scale of HIRES could be in error by $\sim 2 \mathrm{~km} / \mathrm{s}$.

Finally, "local" velocity offsets between the HI $21 \mathrm{~cm}$ and $\mathrm{CI}$ lines within the absorbing galaxies might also contribute to differences in the measured line redshifts. Both $\mathrm{CI}$ and HI $21 \mathrm{~cm}$ absorption are expected to arise in cold gas, and, for both background quasars, a significant fraction of the radio flux density at the HI $21 \mathrm{~cm}$ line frequency arises from a compact core (Kanekar et al.2009). In fact, Q2337-011 has a highly inverted spectrum, indicating that most of the flux density arises from a self-absorbed core (Kanekar et al. 2009). The cold cloud producing $\mathrm{CI}$ absorption against the optical quasar is thus also likely to give rise to HI $21 \mathrm{~cm}$ absorption against the radio core. Assuming that gravity is important for cloud confinement on small scales, Murphy et al. (2003) obtain a velocity dispersion of $\sim 0.1 \mathrm{~km} / \mathrm{s}$ between different species in an individual cloud, which would yield a systematic error of $\sim 3.3 \times 10^{-7}$ in $\Delta X / X$, significantly lower than our statistical errors. We note, however, that larger velocity offsets due to small-scale structure in the absorbing gas cannot formally be ruled out.

\section{DISCUSSION}

Prior to this work, the most sensitive result constraining changes in $X \equiv g_{p} \alpha^{2} / \mu$ using the hyperfine/resonance comparison was that of Tzanavaris et al. (2007). These authors compared redshifts of the deepest absorption in HI $21 \mathrm{~cm}$ and low-ionization metal lines to obtain $\Delta X / X=(6.3 \pm 9.9) \times$ $10^{-6}$ from a sample of nine absorbers at $0.23<z<2.35$. Note that these error estimate do not include systematic effects. Considering only statistical errors, our result, $\Delta X / X=$ $[+6.8 \pm 1.0($ statistical $)] \times 10^{-6}$ over $0<\langle z\rangle<1.46$, is an order of magnitude more sensitive than that of Tzanavaris et al. (2007). Further, the low-ionization metal lines used by Tzanavaris et al. (2007) could also arise in warm HI or ionized gas, and are not necessarily associated with the cold $\mathrm{HI}$ that gives rise to the $\mathrm{HI} 21 \mathrm{~cm}$ absorption. Most of the absorbers used by Tzanavaris et al. (2007) also have complex HI $21 \mathrm{~cm}$ and metal line profiles, and it is not necessary that the deepest absorption in the two types of transitions arises in the same spectral component (Kanekar et al. 2006). This could give systematic errors of $\geq 10 \mathrm{~km} / \mathrm{s}$, far larger than the statistical errors of Tzanavaris et al. (2007), or the systematic errors in the present result.

The comparison between hyperfine and resonance transitions directly probes changes in $X \equiv g_{p} \alpha^{2} / \mu$, and one cannot obtain independent constraints on the individual constants without additional assumptions. Our result gives $2 \times$ $[\Delta \alpha / \alpha]+\left[\Delta g_{p} / g_{p}\right]-[\Delta \mu / \mu]=[+6.8 \pm 1.0($ statistical $) \pm$ 6.7 (systematic) $] \times 10^{-6}$. Note that $6.7 \times 10^{-6}$ is the maximum estimated error due to systematics in the absolute wave- length calibration of the optical spectra, (and not a $1 \sigma$ estimate, as in the case of the statistical error). This implies that $2 \times[\Delta \alpha / \alpha]+\left[\Delta g_{p} / g_{p}\right]-[\Delta \mu / \mu] \geq[+0.1 \pm 1.0] \times 10^{-6}$. The Keck/HIRES result of Murphy et al. (2004) is $[\Delta \alpha / \alpha]=$ $(-5.7 \pm 1.1) \times 10^{-6}$, with $\langle z\rangle=1.75$. Consistency between the two results would require either (1) an additional wavelength calibration error of $\sim 1.7 \mathrm{~km} / \mathrm{s}$ in the CI spectra, which appears unlikely, (2) a "local" velocity offset of $\sim 1.7 \mathrm{~km} / \mathrm{s}$ (and with the appropriate sign) between the HI and CI lines in both absorbers, (3) under-estimated errors in the manymultiplet result [e.g. the distortions in the wavelength scale found by Griest et al. (2010)], or (4) $\left[\Delta g_{p} / g_{p}\right]-[\Delta \mu / \mu] \geq$ $(+1.14 \pm 0.24) \times 10^{-5}$ at $\langle z\rangle=1.46$. In other words, assuming that the errors in one (or both) of the results have not been under-estimated, consistency between the results requires that fractional changes in $\mu$ and/or $g_{p}$ are comparable to those in $\alpha$. Strong constraints are available on fractional changes in $\mu$ at both higher and lower redshifts, $[\Delta \mu / \mu]<1.6 \times 10^{-6}$ at $z \sim 0.685$ (Murphy et al. 2008a) and $[\Delta \mu / \mu]<6.0 \times 10^{-6}$ at $z \sim 2.8$ (King et al. 2008), making it unlikely that $[\Delta \mu / \mu] \sim$ $10^{-5}$ at $z \sim 1.46$. We thus conclude that the present result appears inconsistent with the smaller value of $\alpha$ at $\langle z\rangle \approx 1.75$ found by Murphy et al. (2004), unless fractional changes in the proton gyromagnetic ratio $g_{p}$ are larger than those in $\alpha$ and $\mu$.

In summary, we have detected narrow HI $21 \mathrm{~cm}$ and CI absorption in two absorbers at $z \sim 1.4-1.6$ towards Q0458-020 and Q2337-011, using the Keck telescope, the GMRT and the GBT. The CI and Hi $21 \mathrm{~cm}$ line frequencies have different dependences on the fundamental constants $\alpha, \mu \equiv m_{p} / m_{e}$ and $g_{p}$, allowing us to use the measured CI and $\mathrm{HI} 21 \mathrm{~cm}$ line redshifts to test for putative changes in these constants. Comparing the $\mathrm{HI} 21 \mathrm{~cm}$ and $\mathrm{CI}$ redshifts in the two absorbers yields the result $\Delta X / X=[+6.8 \pm 1.0($ statistical $) \pm$ 6.7 (systematic) $] \times 10^{-6}$ over $0<\langle z\rangle 1.46$, where $X \equiv g_{p} \alpha^{2} / \mu$. This is inconsistent with evidence for a smaller value of $\alpha$ at similar redshifts from the many-multiplet method, unless fractional changes in $g_{p}$ are larger than those in $\alpha$ and $\mu$. Systematic errors in the hyperfine/resonance comparison are currently dominated by errors $(\sim 2 \mathrm{~km} / \mathrm{s})$ in the absolute wavelength calibration of the optical spectra. However, the comparison between HI $21 \mathrm{~cm}$ and $\mathrm{CI}$ lines has a high sensitivity, and it should be possible to significantly reduce systematic effects by the use of new calibration techniques (e.g. laser frequency combs; Steinmetz et al. 2008). Increasing the number of detections of redshifted, narrow HI $21 \mathrm{~cm}$ and CI absorption is thus of much importance.

Some of the data presented herein were obtained at the W.M. Keck Observatory, which is operated as a scientific partnership among the California Institute of Technology, the University of California and the National Aeronautics and Space Administration. The Observatory was made possible by the generous financial support of the W.M. Keck Foundation. We thank the staff of the GMRT and the GBT for help during these observations. The GMRT is run by the National Centre for Radio Astrophysics of the Tata Institute of Fundamental Research. The National Radio Astronomy Observatory is operated by Associated Universities, Inc, under cooperative agreement with the NSF. NK acknowledges support from the Max-Planck Society, and from the Department of 


\section{REFERENCES}

Berengut, J. C., Flambaum, V. V., \& Kozlov, M. G. 2006, Phys. Rev. A, 73, 012504

Carilli, C. L., Menten, K. M., Stocke, J. T., Perlman, E., Vermeulen, R., Briggs, F., de Bruyn, A. G., Conway, J., \& Moore, C. P. 2000, Phys. Rev. Lett., 85, 5511

Chengalur, J. N. \& Kanekar, N. 2003, Phys. Rev. Lett., 91, 241302

Cowie, L. L. \& Songaila, A. 1995, ApJ, 453, 596

Dessauges-Zavadsky, M., Calura, F., Prochaska, J. X., D’Odorico, S., \& Matteucci, F. 2004, A\&A, 416, 79

Dzuba, V. A., Flambaum, V. V., \& Webb, J. H. 1999, Phys. Rev. Lett., 82, 888

Essen, L., Donaldson, R. W., Bangham, M. J., \& Hope, E. G. 1971, Nature, 229,110

Flambaum, V. V. \& Kozlov, M. G. 2007, Phys. Rev. Lett., 98, 240801

Griest, K., Whitmore, J. B., Wolfe, A. M., Prochaska, J. X., Howk, J. C., \& Marcy, G. W. 2010, ApJ, 708, 158

Jenkins, E. B. \& Tripp, T. M. 2001, ApJS, 137, 297

Kanekar, N. 2008, Mod. Phys. Lett. A, 23, 2711

Kanekar, N., Carilli, C. L., Langston, G. I., Rocha, G., Combes, F. Subrahmanyan, R., Stocke, J. T., Menten, K. M., Briggs, F. H., \& Wiklind, T. 2005, Phys. Rev. Lett., 95, 261301

Kanekar, N., Chengalur, J. N., \& Ghosh, T. 2004, Phys. Rev. Lett., 93, 051302

Kanekar, N., Prochaska, J. X., Ellison, S. L., \& Chengalur, J. N. 2009, MNRAS, 396, 385

Kanekar, N., Subrahmanyan, R., Ellison, S. L., Lane, W. M., \& Chengalur, J. N. 2006, MNRAS, 370, L46

King, J. A., Webb, J. K., Murphy, M. T., \& Carswell, R. F. 2008, Phys. Rev. Lett., 101, 251304
Levshakov, S. A., Centurión, M., Molaro, P., D’Odorico, S., Reimers, D., Quast, R., \& Pollmann, M. 2006, A\&A, 449, 879

Marciano, W. J. 1984, Phys. Rev. Lett., 52, 489

Morton, D. C. 2003, ApJS, 149, 205

Murphy, M. T., Flambaum, V. V., Muller, S., \& Henkel, C. 2008a, Science, 320,1611

Murphy, M. T., Flambaum, V. V., Webb, J. K., Dzuba, V. V., Prochaska, J. X., \& Wolfe, A. M. 2004, in Lecture Notes in Physics, Vol. 648, Astrophysics, Clocks and Fundamental Constants, ed. S. G. Karshenboim \& E. Peik (Berlin: Springer-Verlag), 131

Murphy, M. T., Webb, J. K., \& Flambaum, V. V. 2003, MNRAS, 345, 609

Murphy, M. T., Webb, J. K., \& Flambaum, V. V. 2008b, MNRAS, 384, 1053

Murphy, M. T., Webb, J. K., Flambaum, V. V., Churchill, C. W., \& Prochaska, J. X. 2001, MNRAS, 327, 1223

Pettini, M., Boksenberg, A., Bates, B., McCaughan, R. F., \& McKeith, C. D. 1977, A\&A, 61, 839

Prochaska, J. X. \& Wolfe, A. M. 1997, ApJ, 487, 73

Srianand, R., Chand, H., Petitjean, P., \& Aracil, B. 2007, Physical Review Letters, 99, 239002

Srianand, R., Petitjean, P., Ledoux, C., Ferland, G., \& Shaw, G. 2005, MNRAS, 362, 549

Steinmetz, T., Wilken, T., Araujo-Hauck, C., Holzwarth, R., Hänsch, T. W. Pasquini, L., Manescau, A., D’Odorico, S., Murphy, M. T., Kentischer, T., Schmidt, W., \& Udem, T. 2008, Science, 321, 1335

Tzanavaris, P., Webb, J. K., Murphy, M. T., Flambaum, V. V., \& Curran, S. J 2007, MNRAS, 374, 634

Uzan, J.-P. 2003, Rev. Mod. Phys, 75, 403

Wolfe, A. M., Broderick, J. J., Condon, J. J., \& Johnston, K. J. 1976, ApJ, 208, L47 